protein or $1000 \mathrm{ng} / \mathrm{ml}$ of human IgG1 as a control for $12 \mathrm{~h}$. Gene expressions were detected by microarray assay.

Results: Microarray data analysis revealed that DR3 up-regulated or downregulated the expression of various genes in RA-FLS (Figure). The function of regulated genes included protein-L-isoaspartate (D-aspartate) O-methyltransferase activity, carboxyl-O-methyltransferase activity, protein carboxyl O-methyltransferase activity, regulation of cilium assembly, O-methyltransferase activity, regulation of plasma membrane bounded cell projection assembly, regulation of cell projection assembly, regulation of organelle assembly, protein methyltransferase activity, and S-adenosylmethionine-dependent methyltransferase activity. The most up-regulated 2 genes by DR3 were KIAA1109 (KIAA1109), and adhesion $G$ protein-coupled receptor A3 (ADGRA3). The most down-regulated 2 genes by DR3 were RNA exonuclease 2 (REXO2), and family with sequence similarity 120A (FAM120A)

Conclusion: In this study, we first revealed the expression profiles of genes regulated by DR3 in RA-FLS. KIAA1109/TENR/IL2/IL21 gene is strongly associated with RA in European descent populations [3]. ADGRA3 is a member of G protein-coupled receptors (GPCRs). GPCRs associates with the regulation of cytoskeletal organization, the cell adhesion and migration, cell proliferation and apoptosis, and cell differentiation [4]. Loss of REXO2 affects cell growth and morphology [5], and REXO2 was identified as a target gene for inflammatory bowel disease-associated variants [6]. FAM120A regulates activity of Src kinase to protect cells from oxidative stress-induced apoptosis [7]. DR3 regulates the gene expressions of various key molecules in RA-FLS and may affect the pathogenesis of RA by regulating gene expression of RA-FLS.

References:

[1] Takami N. et al., Arthritis Rheuma. 2006;54:779-787.

[2] Fukuda K. et al., Biomed Rep. 2019;1:1-5.

[3] Teixeira VH. et al., Arthritis Research \& Therapy. 2009;11:R45.

[4] Hamann J. et al., Pharmacol Rev. 2015;67:338-367.

[5] Bruni F, et al., PLoS One. 2013;8:e64670.

[6] Hulur I, et al., BMC Genomics. 2015;16:138.

[7] Tanaka M. et al., Mol Cell Biol. 2009;29:402-413.

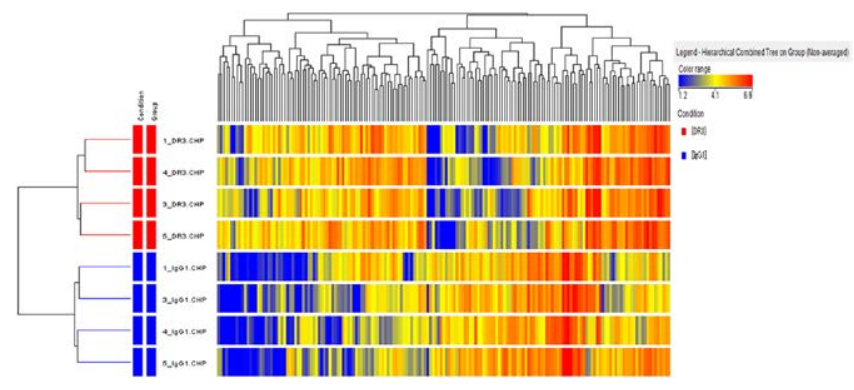

Disclosure of Interests: None declared

DOI: 10.1136/annrheumdis-2020-eular.1466

\section{AB0091 PD-1 AND GAL3 REGULATE OSTEOCLAST DEVELOPMENT IN RHEUMATOID ARTHRITIS}

S. R. Greisen ${ }^{1,2}$, M. A. Nielsen ${ }^{1,2}$, M. Hvid ${ }^{3}$, B. Deleuran ${ }^{1,2} .^{1}$ Aarhus University, Dept. of Biomedicine, Aarhus, Denmark; ${ }^{2}$ Aarhus University Hospital, Dept. of Rheumatology, Aarhus, Denmark; ${ }^{3}$ Aarhus University, Dept of Clinical Medicine, Aarhus, Denmark

Background: Bone erosions in rheumatoid arthritis (RA) is a major complication. Despite improved treatment, erosions still occur and progress. Therefore, a continuous investigation of the interplay between bone regulation and immune activity is needed.

Co-inhibitory receptors, like CTLA-4, participate in modulating osteoclast activity ${ }^{1}$, and blocking these receptors in cancer treatment results in autoimmune disease ${ }^{2}$. Programmed death 1 (PD-1) is a central co-inhibitory receptor, also present in a soluble (s) form. We have previously shown that SPD-1 is associated with disease activity and reduced bone erosions in $R A^{3}$. PD-1 and its ligands are glycosylated and the glycosylation affects signaling through the PD-1 pathway ${ }^{4,5}$. Galectins (GAL) bind to specific glycosylation patterns on glycoproteins by their carbonrecognition domain. Gal-3 can bind to multiple immune receptors shaping the immunological response ${ }^{6}$.

Objectives: We aimed to investigate if PD-1 and GAL-3 modulate osteoclastogenesis in RA.

Methods: Plasma and synovial fluid (SF) samples were collected from patients with chronic (c) RA (>8 years of disease, $n=14$ ) SF and blood samples were obtained when patients presented with disease flare. Soluble PD-1 and GAL-3 were investigated by ELISA. GAL-3:PD-1 complexes were captured in an optimized ELISA using both GAL-3 and PD-1 antibodies.
Surface plasmon resonance was used to evaluate on the binding properties between GAL-3 and PD-1, on a Biacore 3000. Lactose was used to block the potential binding. Cells from the synovial fluid (SFMC) were differentiated into osteoclasts with M-CSF and RANKL, recombinant human (rh) PD-1 and rhGAL-3 were added and osteoclast formation evaluated by TRAP in the supernatant.

Results: Soluble PD-1 and GAL-3 were present in both plasma and SF from cRA patient, and the ratio (PD-1/GAL-3) was increased in SF. PD-1:GAL-3 complexes were detected in both plasma and SF, with the highest concentration in SF. The binding between PD-1 and GAL-3 was confirmed by surface plasmon resonance analyses, with an estimated $\mathrm{Kd}$ of $5 \mathrm{uM}$. Binding could be blocked by addition of lactose, confirming the binding to be glycan dependent. In SFMC osteoclast cultures, rhPD-1 and rhGAL-3 slightly decreased osteoclast formation evaluated by TRAP. However, combining rhPD-1 and rhGAL3 further potentiated the reduction in osteoclast formation by $37 \%$.

Conclusion: We confirm glycan depended binding between the co-inhibitory receptor PD-1 and GAL-3. Both SPD-1 and Gal-3, and the PD-1:GAL-3 complexes, are upregulated in the inflamed joint at site of erosions. In vitro RA culture demonstrates that GAL-3 potentiate the functions of PD-1 and reduces osteoclastogenesis. These findings indicate that the binding between Gal-3 and PD-1 could provide a novel target to control erosions in RA. Future in vivo studies on this interaction is needed.

\section{References:}

[1] Axmann, R. et al. CTLA-4 directly inhibits osteoclast formation. Ann Rheum Dis 67, 1603-1609 (2008).

[2] Friedman, C. F., Proverbs-Singh, T. A. \& Postow, M. A. Treatment of the Immune-Related Adverse Effects of Immune Checkpoint Inhibitors. JAMA Oncol 2, 1346-8 (2016).

[3] Greisen, S. et al. Increased soluble programmed death-1 (sPD-1) is associated with disease activity and radiographic progression in early rheumatoid arthritis. Scand J Rehabil Med 43, 101-108 (2013).

[4] Verdura, S. et al. Resveratrol targets PD-L1 glycosylation and dimerization to enhance antitumor T-cell immunity. Aging (Albany NY) 12, 8-34 (2020).

[5] Wang, M. et al. Identification of a monoclonal antibody that targets PD-1 in a manner requiring PD-1 Asn58 glycosylation. Com Biol 1-10 (2019). doi:10.1038/s42003-019-0642-9

[6] de Oliveira, F. L. et al. Galectin-3 in autoimmunity and autoimmune diseases. Exp Biol Med (Maywood) 240, 1019-1028 (2015).

Disclosure of Interests: None declared

DOI: 10.1136/annrheumdis-2020-eular.5375

\section{AB0092 \\ FIBRIN ADHESION IS A PANNUS-INDEPENDENT MECHANISM OF CARTILAGE DEGENERATION IN RHEUMATOID ARTHRITIS}

T. Hügle ${ }^{1}$, S. Nasi ${ }^{2}$, D. Ehirchiou ${ }^{1}$, A. So ${ }^{3}$, N. Busso ${ }^{3} .{ }^{1}$ University Hospital Lausanne (CHUV), Lausanne, Switzerland; ${ }^{2}$ University Hospital Münster, Institute of Musculoskeletal Medicine, Münster, Germany; ${ }^{3}$ University Hospital Lausanne (CHUV), Rheumatology, Lausanne, Switzerland

Background: Current concepts of cartilage destruction in inflammatory arthritis include pannus infiltration by inflamed synovial tissue as well as direct detrimental effects of inflammatory cytokines and proteinases. Fibrin maintains chronic inflammatory processes in arthritis but has never been shown to be directly involved in cartilage damage occurring in rheumatoid arthritis (RA).

Objectives: To investigate fibrin-mediated cartilage degradation and the possible underlying mechanisms in arthritis.

Methods: Human cartilage samples were obtained from patients with RA undergoing joint replacement and investigated by H.E. and immunohistochemistry for cartilage damage and fibrin deposition. Cartilage explants from RA patients were incubated in vitro with autologous synoviocytes and assessed by immunohistochemistry for cell-adhesion and colocalization with fibrin. Experimental RA was studied in the RA murine model of adjuvant-induced arthritis (AIA), in wildtype (WT) and fibrinogen deficient $\left(\mathrm{Fg}^{-1-}\right)$ mice. Cartilage damage and chondro-synovial adhesion were analyzed by safranin-O staining and fibrin deposition by immunohistochemistry. Fibrinogen expression ( $F g a, F g \beta, F g \gamma$ ) was studied in murine primary chondrocytes by qRT-PCR. Cartilage explants were stained with alizarin-red staining and assessed for colocalization of calcific deposits and fibrin. Calcification of murine primary chondrocytes stimulated with secondary calciprotein particles (CPP) and treated with purified human plasma fibrinogen $(100 \mu \mathrm{g} / \mathrm{ml})$ was assessed by alizarin red staining and gene expression for chondrocytic differentiation (Agg, Coll2, Coll10, Sox9, Runx2), calcification (Alpl, Ank, Anx5, Pc1, Pit1, Pit2), and extracellular matrix remodeling (Adamts4, Adamts5, Mmp3, Mmp13, Comp) by qRT-PCR.

Results: Abundant fibrin deposition on cartilage co-localized and positively correlated with cartilage damage in knee joints of patients with RA. In the AIA model 
absence of fibrin deposition in $\mathrm{Fg}^{-/-}$mice was accompanied by significantly lower synovial inflammation, chondro-synovial adhesion and cartilage damage than in WT mice. Chondro-synovial adhesion correlated with cartilage damage in the WT and led to apparent mechanical stripping of the superficial cartilage, whilst this phenomenon was not observed in the $\mathrm{Fg}^{-/-}$mice. In vitro, autologous RA synoviocytes adhered to cartilage explants exclusively in the presence of fibrin deposition. Fibrinogen chains were not expressed by primary chondrocytes, indicating passive deposition from synovial fluid or tissue. In human RA cartilage explants, we found colocalization and a significant positive correlation between fibrin and calcific deposits. Fibrinogen caused exacerbated calcification in CPPtreated primary murine chondrocytes and induction of genes involved in chondrocyte calcification (Pc1, Pit1). Cartilage-oligomeric matrix protein (Comp) gene was also highly induced suggesting a pro-catabolic role of fibrinogen.

Conclusion: Fibrin deposition is an active trigger of cartilage degeneration in RA via induction of chondro-synovial adhesion (mechanical aspect) and induction of calcification (catabolic aspect). Newer therapeutic approaches may not merely focus on fibrinolysis but protect cartilage from fibrin-induced adhesion or calcification e.g. by fibrin-targeted immunotherapy.

Disclosure of Interests: Thomas Hügle Grant/research support from: Abbvie, Novartis, Consultant of: Abbvie, Pfizer, Novartis, Roche, Lilly, BMS, Sonia Nasi: None declared, Driss Ehirchiou: None declared, Alexander So Consultant of: Sobi, Grünenthal, Nathalie Busso: None declared

DOI: 10.1136/annrheumdis-2020-eular.5737

\section{\begin{tabular}{l|l}
\hline AB0093 & PORPHYROMONAS GINGIVALIS - PERIODONTAL
\end{tabular} PATHOGEN WITH POTENTIAL ROLL IN RHEUMATOID ARTHRITIS}

P. Selimov ${ }^{1}$, E. Firkova ${ }^{2}$, L. Damjanovska-Krstikj ${ }^{3}$, A. Batalov ${ }^{1}$, A. Maneva ${ }^{4}$, R. Karalilova ${ }^{1}$, G. Delcheva ${ }^{4}$, K. Stefanova ${ }^{4}$, T. Stankova ${ }^{4} .{ }^{1}$ Medical University of Plovdiv, Department of Propaedeutics of Internal Diseases, Plovdiv, Bulgaria; ${ }^{2}$ Medical University of Plovdiv, Department of Periodontology and Oral Diseases, Plovdiv, Bulgaria; ${ }^{3}$ University Rheumatology Clinic Skopje, Skopje, Macedonia, Rep. of; ${ }^{4}$ Medical University of Plovdiv, Department of Chemistry and Biochemistry, Plovdiv, Bulgaria

Background: In recent literature, a strong association between periodontal disease (PD) and rheumatoid arthritis (RA) has been reported. PD is a common, progressive inflammatory disease, initiated by a bacterial infection that engages the supporting structures of the teeth and leads to tooth loss. A number of common features have been identified between PD and RA.One of the most important associations is the process of citrullination, which is caused by the production of PG specific enzyme so called Porphyromonas Gingivalis Peptydil Deminase (PPAD)

Objectives: The aim of the study was to show the incidence of PG in RA patients, and to compare it with patients with osteoarthritis $(\mathrm{OA})$ and healthy controls $(\mathrm{HC})$ and to evaluate the possible correlation between the presence of PG in patients with RA and the positivity of anti CCP and anti MCV antibodies in RA patients.

Methods: The study included 30 patients with RA which fulfilled RA classification criteria from 2010, 26 patients with osteoarthritis (OA) and 24 healthy controls. All participants were genetically analyzed for the presence of $P G$ by Chelex®100 method and polymerase chain reaction (PCR), by isolating amplified sequences of DNA in a sub gingival biofilm taken from the deep periodontal pockets. The presence of anti CCP and anti MCV autoantibodies was detected in the sera of RA patients with ELISA test.

Results: The average ages of the patients in the 3 groups were as follows -51 years for RA, 52 for OA and 58 years for $\mathrm{HC}$. Seventy two percent of RA patients were females.

Significantly higher levels of $P G$ were found in the periodontal pockets of.RA patients.

Eighty percent of RA patients ( $80 \%$ or 24 RA patients) were PG positive in comparison with $35 \%$ of $O A$ patients and $2 \%$ healthy controls.

Of the PG-positive RA patients, $83 \%$ had positive and $17 \%$ had anti-CCP negative test, while of the PG-negative patients, a positive anti-CCP test was present in 33\% and a negative anti-CCP test was present in 67\%.Accordingly, in PG-positive RA patients positive anti-MCV test was present in $79 \%$ and negative anti-MCV test was present in $21 \%$, and in PG-negative RA patients anti-MCV test was positive in $17 \%$ and negative in $83 \%$ patients.
Table 1.

\begin{tabular}{|c|c|c|c|c|}
\hline Patient's groups & RA & OA & $\mathrm{HC}$ & Significance \\
\hline Porphyromonas gingivalis positive & $24(80 \%)$ & $9(35 \%)$ & $7(29 \%)$ & $\mathrm{RA} \rightarrow \mathrm{OA}$ \\
\hline Porphyromonas gingivalis negative & $6(24 \%)$ & $17(65 \%)$ & $17(71 \%)$ & $\begin{array}{c}(p<0.0001) \\
R A \rightarrow H C \\
(p<0.0001) \\
O A \rightarrow H C \\
(p 0,65)\end{array}$ \\
\hline Total & 30 & 26 & 24 & \\
\hline
\end{tabular}

Conclusion: The results of our study indicate that $P G$ is found more frequently in periodontal pockets of patients with rheumatoid arthritis, which implies the important role of oral microbioma in RA pathogenesis, treatment and prevention. References:

[1] Mikuls T. R., Thiele G. M., Deane K. D. et al. (2012). Porphyromonas gingivalis and disease-related autoantibodies in individuals at increased risk of rheumatoid arthritis. Arthritis \& Rheumatism, 64(11), 3522-3530.

[2] Mikuls T.R., Payne J.B., Yu F. et al. Periodontitis and Porphyromonas gingivalis in patients with rheumatoid arthritis. Arthritis Rheumatol. 2014; 66(5): 1090-1100

Disclosure of Interests: Pavel Selimov: None declared, Elena Firkova: None declared, Ljubinka Damjanovska-Krstikj Grant/research support from: Roche, Speakers bureau: Pfizer, Anastas Batalov: None declared, Ana Maneva: None declared, Rositsa Karalilova: None declared, Ginka Delcheva: None declared, Katia Stefanova: None declared, Teodora Stankova: None declared DOI: 10.1136/annrheumdis-2020-eular.6300

\section{AB0094 FUNCTIONAL TREG CELLS MAY BE CONVERTED INTO T EFFECTOR PHENOTYPE ON EXPOSURE TO INFLAMMATORY MILIEU IN RHEUMATOID ARTHRITIS (RA) SYNOVIAL FLUID: IN-VITRO STUDY}

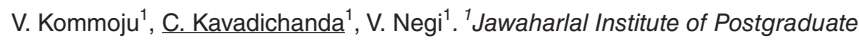
Medical Education and Research, Clinical Immunology, Puducherry, India

Background: The debate on functional versus numerical difference in $T$ regulatory cell population among patients with Rheumatoid arthritis (RA) is not clear. Tregs expressing Inflammatory subset phenotype markers, such as Th1(cxcr3, Tbet) and Th17(ccr6,Rorr) are reported. (1) Though the reported numbers of synovial fluid tregs are higher in RA, (2) the fate of Tregs on entering the synovial inflammatory milieu from peripheral blood (PB) has not yet been investigated.

Objectives: To compare Treg frequencies in PB and synovial fluid between osteoarthritis (OA) and RA

To compare cytokine levels in PB and SF between OA and RA

To study the effect of autologous synovial fluid on RA and OA Treg isolated form peripheral blood

Methods: The Peripheral Blood (PB) and synovial fluid (SF) of RA $(n=80)$ and $O A(n=30)$ patients were analyzed for CD4+T-cell subset frequencies and phenotypes by flow cytometry. Cytokine concentrations in plasma and SF were measured by cytometric bead array. Tregs from 5 RA-PB and 5 OA-PB were isolated and cultured in autologous synovial fluid for $24 \mathrm{hrs}$. Phenotypic expression of Th1 and Th17 chemokines on the cell surface were analyzed by flow cytometry and expression levels of T-bet, ROR $\gamma$ and FOXP3 in those Treg cells were measured with quantitative real-time PCR (RT-qPCR).

Results: The PB and SF frequencies of Th1, Th17 and Tregs are shown in Table 1. The pro-inflammatory cytokines were high in the plasma and SF of RA but the anti-Inflammatory cytokines were similar (Fig 1.A\&B). Treg cells were isolated from RA and OA PB and cultured in autologous SF for 24 hrs. RA Treg showed increased cell surface expression of CXCR3+ and CCR6+ (Fig 1C) and there was no difference in OA Treg. Gene expression studies showed an increased expression of T-bet, ROR $y$ and decreased expression of Foxp3 in RA Tregs while there was no difference in OA Tregs before and after in-vitro culture (Fig 1D). 